\title{
Simulation Analysis Software Correction Method
}

\author{
Prasjendra Kuehar $^{1}$, Anil Reaurve ${ }^{2}$, Shaliender Shkehawat ${ }^{3}$ \\ ${ }^{1}$ Department of Computer Science and Engineering, Walchand College of Engineering (An Autonomous Institute), Sangli, India \\ prasad.kumbhar@walchandsangli.ac.in \\ ${ }^{2}$ Department of Computer Science and Engineering, Walchand College of Engineering (An Autonomous Institute), Sangli, India \\ anil.surve@walchandsangli.ac.in \\ ${ }^{3}$ Calibration Company, India \\ shailender.shekhawat@gmail.com
}

\begin{abstract}
Performance is one of the key factors in web application success. Nowadays, users Expect a steady availability and prompt response after their action. To meet with that expectation, our goal was to measure efficiency and effectiveness Calibration software. Calibration software is web-based calibration management tool that helps you maintain and control your calibration organization. Also manage the calibration of all measurement devices for the specified business location and set up a calibration work schedule for technicians. As part of this research used the JMeter tool and the Performance Monitor Tool to perform these tests. Study argue that the efficiency of calibration software can be significantly increased. In this research analyzed non-functional CTQs, described our approach for testing and measuring their functionality, and presented our results. Finally, this research calculates the total efficiency of each JMeter test case, using the weight given to each performance indicator measured, including the time of technology implementation.
\end{abstract}

Keywords: Performance Monitor, JMeter Scripts, Non-Functional CTQs, Calibration

\section{Introduction}

This is the next generation calibration and asset management software that provides full visibility of all assets, references standards and plant resources. This software effectively integrates people with the organization's pressure calibrators and controllers to provide a holistic calibration solution. It allows easy, safe and effective maintenance, it helps to streamline the process by enhancing efficiency and avoiding human errors. The web browser-based design of calibration software allows you to access data anytime, anywhere and eliminate the need to maintain and manage paper-based records.

1) Maintaining Simple, Smart, Scalable Calibration software offers the flexibility you need - calibration Management software is a web-based architecture that allows users to simply log in web browser from any location. Calibration software reduces calibration time and provides automation and integration capabilities cost once installed, it can be easily calculated for a large number of users or devices to meet your growing calibration needs [1].

2) Compliant and audit-ready: It offers paperless, automated workload schedules and management, extensive control over calibration workflow and data, easy access to historical calibrations, and ability to generate ports in customized formats [1].

3) Calibration made smart: This allows the functionality of the historical trending module assets for maintenance from time to time. Calibration software provides strong solutions to error analysis and historical trending. Then understand the deviations and errors used to apply the correct procedure corrective action and preventive action (CAPA) to reduce calibration errors improving quality [1].
Web application gives users user friendly interface to send the web request and wait for response from the server. Server calls the services of the application. It is very mandatory to test the services from end to end. As web application use interface of loosely coupled architecture it is necessary to test services and interfaces. There are chances of performance issues because web application uses web services at the back end to fulfil the user requests. That is why a tester must be aware of all the functionality and behaviour of the application [6] [7]. This paper will clarify such two testing devices JMeter and Performance Monitoring Tools [9] [10]. Testing is a cycle of examination of bugs and blunders from the product application. Execution, making of experiments for any climate in the application or administration is overseen by test apparatuses. By utilizing some testing devices, for example, execution testing instrument and JMeter apparatus this paper will expound execution testing, practical testing and dependability testing [5].

Performance testing is yet another type of automated testing which tells the testers that the software or the application will work correctly under an expected condition. Reliability Testing is one more kind of mechanized testing which tells the analyzers that the product or the application will work accurately under a normal condition. [8].

\section{Literature Survey}

Traditional calibration process: - The 8-step process highlights the traditional, paper-based calibration process. This is highly inefficient, labour-intensive and involves manual data storage which is time-consuming and errorprone. 


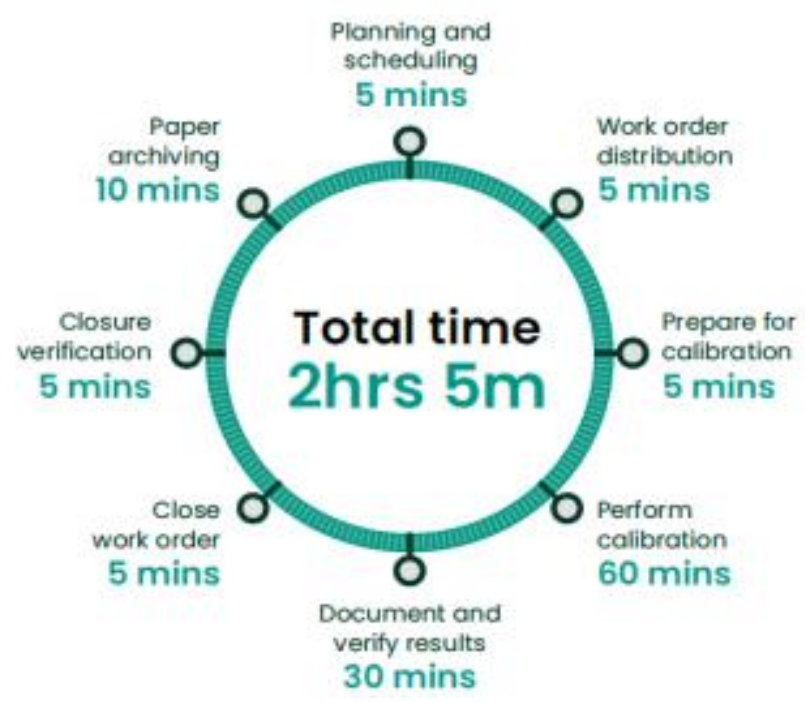

Figure 1: Traditional calibration process.

Less Efficient - The 8-step process takes over 2 hours to complete, due to the intensive preparation and verification of results required.

The new improved calibration process: - By utilizing the Calibration and Asset management software, to automate the calibration process and removing the need for manual data entry and storage, your process can be more efficient, saving you time and money.

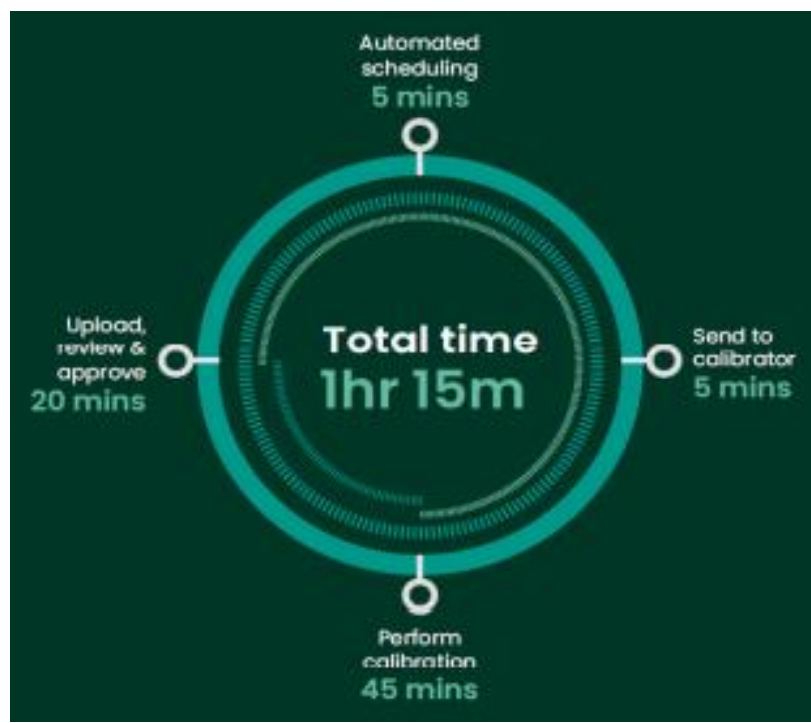

Figure 2: Improved calibration process.

Up to 40 percent faster - Calibration and Asset Management software can complete a calibration 40 percent faster than paper-based calibration. As shown in fig 2.

\section{Research Methodology}

Phase I Performance testing is a type of software testing that ensures system applications perform properly under their expected workload. It runs a testing technique and determining system performance in terms of internal sensitivity, reaction and stability in specific work load [10]. These 12 stages perform the performance testing in a calibration software project, as shown in figure 3 .

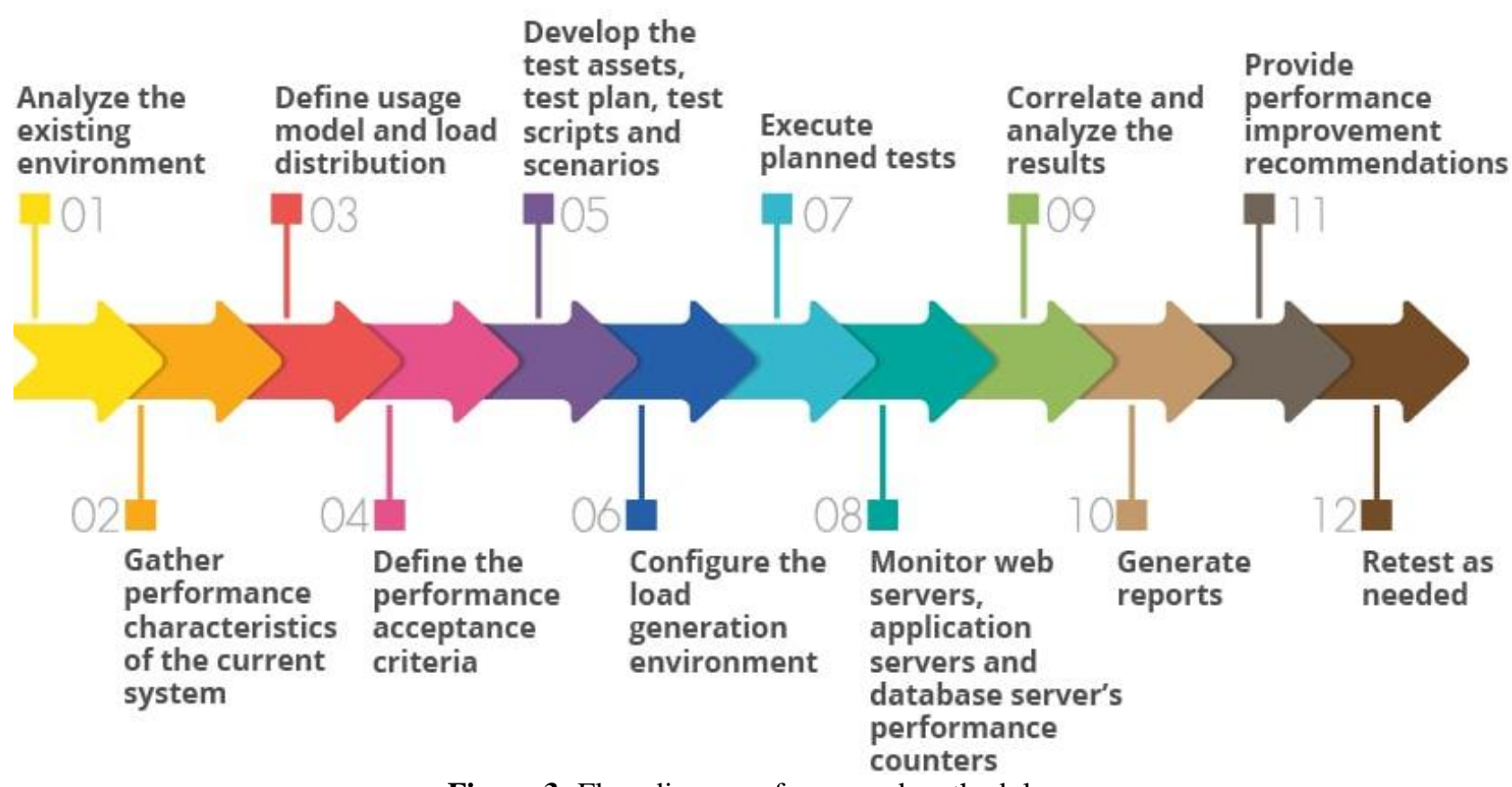

Figure 3: Flow diagram of proposed methodology 
a) Analyze the existing environments: In the existing environment, to overcome the discrepancies implementation is done. Effectiveness of the implementation in current environment have to be checked.

b) Gather performance characteristics of the current system Gathered all required information about performance and the factor present in the performance need to be checked.

c) Define usage model and load distribution Product should have an availability of 99 percentage over 1-year interval. Typical use case for 1 year - For 5000 devices in an enterprise.

d) Define the performance acceptance criteria Application must be run under Wei-bull $2 \mathrm{p}$ model criteria. e) Develop the test assets, test plan, test scripts and scenarios in the system of generated load and perform the $100 \mathrm{~K}$ calibration with 20 concurrent users using JMeter tools. There are different components of JMeter.

JMeter Tools- Apache JMeter is an" open-source testing instrument" created by" Apache Software Foundation (ASF)". JMeter's primary capacity is to stack test customer/worker. Besides, JMeter is utilized in relapse testing by creating test scripts. JMeter gives offline detailing of test outcomes [7]. JMeter test reports are shown in fig 4 .

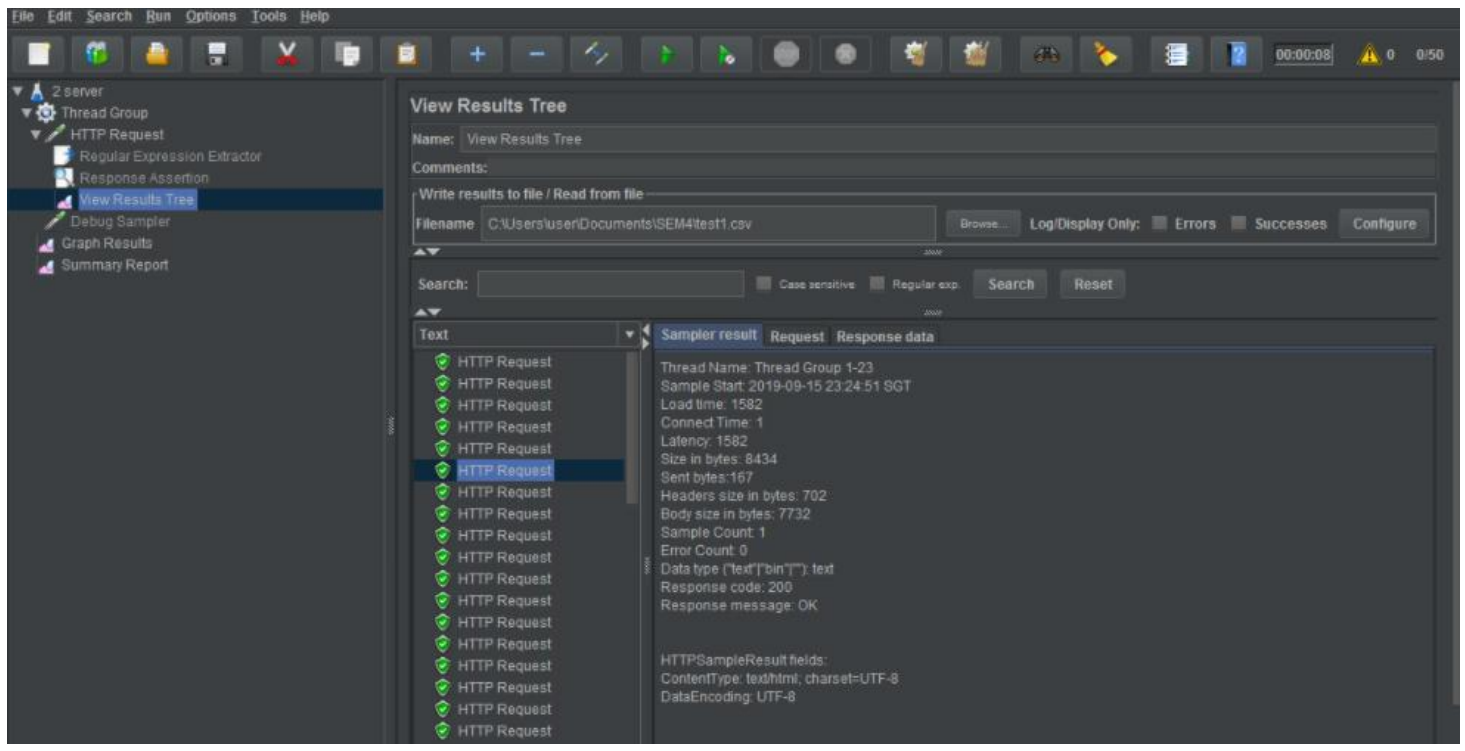

Figure 4: JMeter test reports

f) Configure the load generation environments in the system created sample load on application server and generated $100 \mathrm{k}$ data in database using JMeter script. This data is given in plant, location, sub-location, tag, device, ranges, procedure, routine, users, groups, permission and documents [14]. Connection of JMeter Tools- As shown in Fig 5.

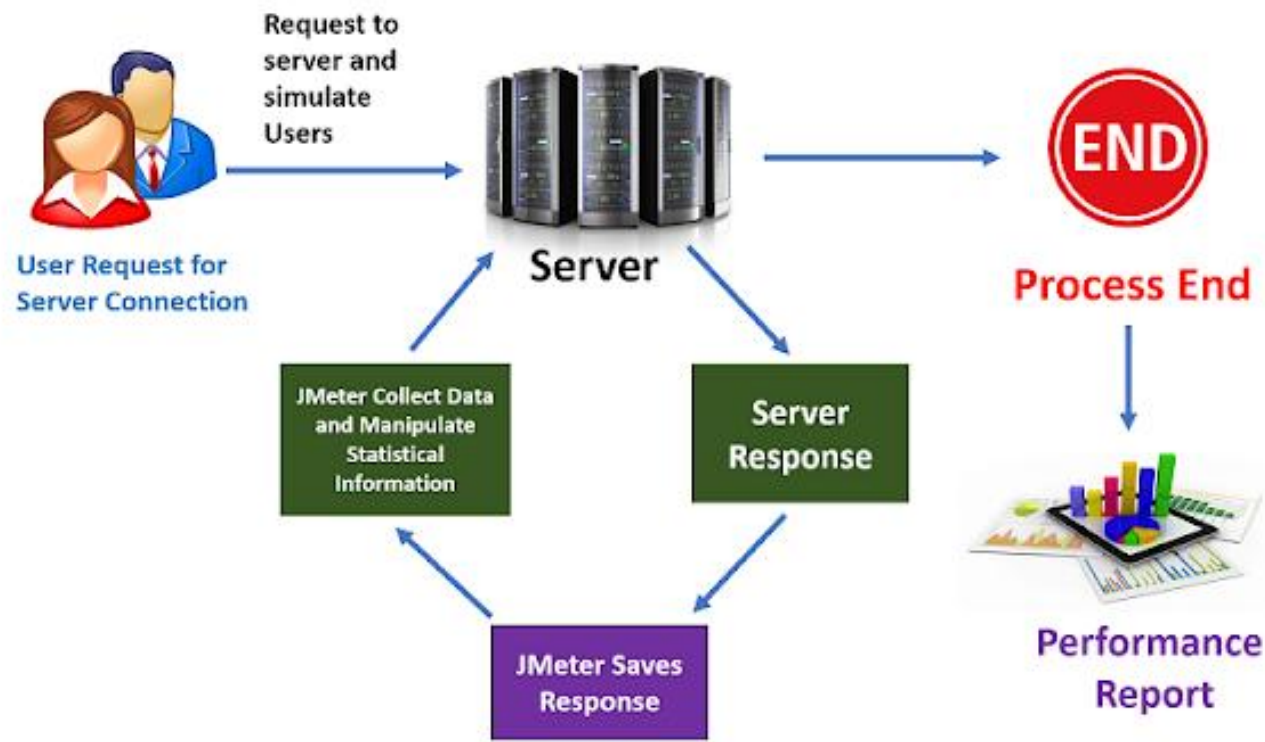

Figure 5: Connection of the proposed system 
g) Execute planned tests Executed the JMeter scripts in calibration software in following order - User Management, Enterprise Creation, Complete job creation Loop, Routine Procedures creation Loop, Routine Procedures Link with Devices and Ranges, Technician and Approver creation and Assign Job, Result Creation MANUAL Calibration, Result Creation SWITCH Calibration and Document Management.

\section{Phase II}

a) Monitor web server, application server and database server's performance counter Monitoring the application and database server from following things - Handle Count, Virtual Bytes, Private Bytes, Thread Count and Working Set this counter start for monitoring a system [15] [16]. Performance Monitor Tools- As shown in Fig 6

b) Correlate and analyze the results Executed system nonfunctional CTQ excel file and analyzed the results, From the results this research understands our system runs properly or not.

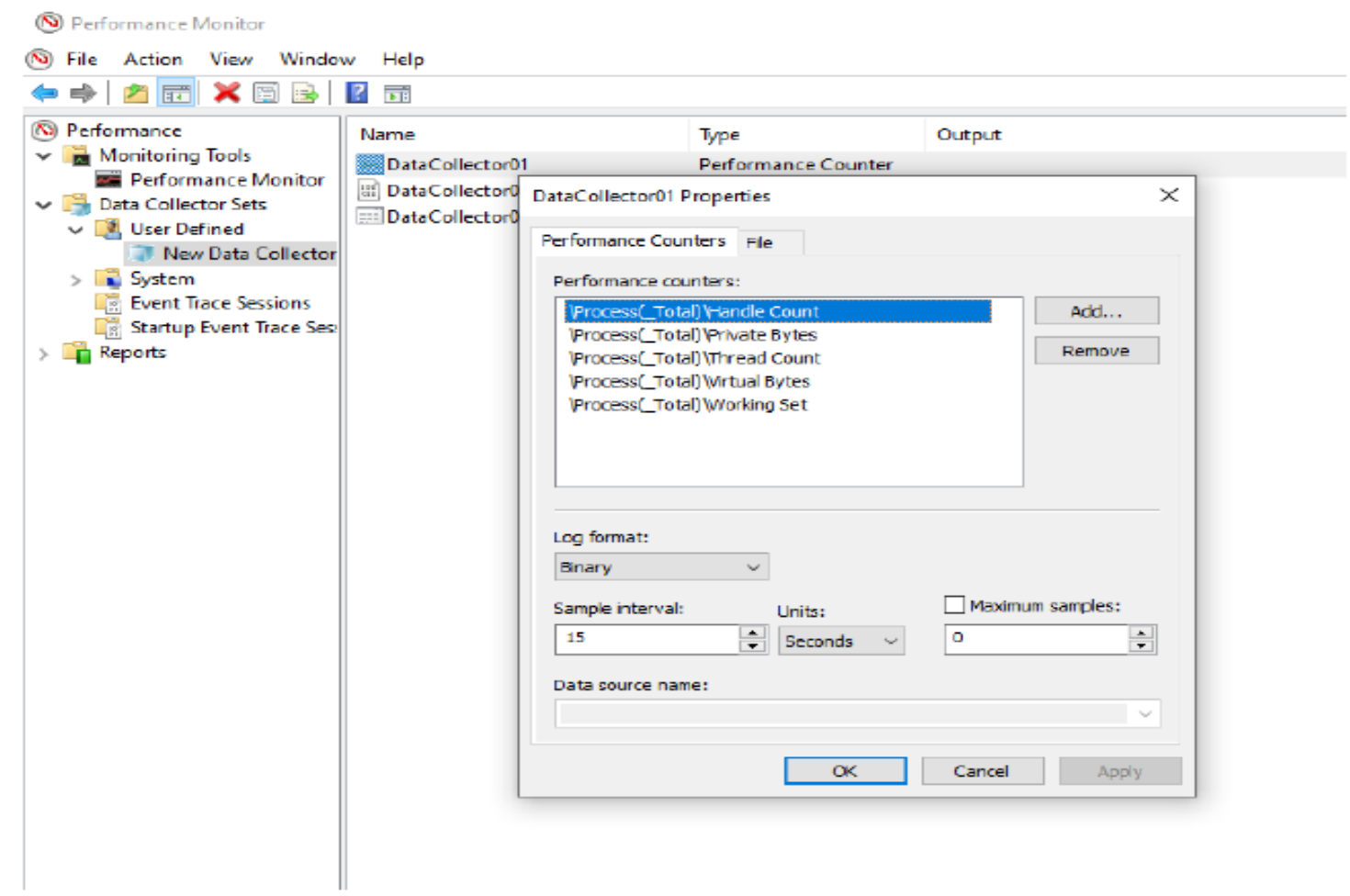

Figure 6: Counter set for performance monitoring

c) Generate reports in this research generated a report and check the reliability of the counter set in the performance monitor tool and represent it graphically [16]. K. Provide performance improvements recommendations This research provides us the recommendations on basis of the results that obtained from the counters [12].

\section{Results and Discussion}

According to this study, this research has given expected results. The system has completed the minimum calibration process as well as the traditional calibration process gives more functionality with less cost. This research reduces the requirement of manpower and paper-based calibration processes. In these attempts results are accurate and specific for the devices and instruments. This study verifies that our system is working on maximum capacity using the JMeter tool. It has analyzed that the system works properly using a set of counters in the performance monitor tool. Calculated Prediction Value Up to 365 Days: In this study calculated the forecast counter value from 21 days of observation to 365 days. The overall condition of a straight line is $y=m x+$ $\mathrm{c}$, where $\mathrm{m}$ is the slope, and $\mathrm{y}=\mathrm{c}$ is the worth where the line cuts the $y$-hub. This number $\mathrm{c}$ is known as the block on the y-pivot." $\mathrm{Y}=\mathrm{mx}+\mathrm{c}$ " this equation helps to find or prediction of data up to 365 days. 


\begin{tabular}{|c|c|c|c|c|c|c|c|c|}
\hline \multirow{3}{*}{$\begin{array}{l}\text { This EQ generted under the performance } \\
\text { Moinitor } 21 \text { Days Observation }\end{array}$} & & & \multicolumn{2}{|l|}{ Dummy Data } & \multirow[b]{3}{*}{$\begin{array}{l}\text { Projected } 90 \\
\text { Days from Eq }\end{array}$} & \multirow[b]{3}{*}{$\begin{array}{l}\text { Projected } 180 \\
\text { Days from Eq }\end{array}$} & \multirow[b]{3}{*}{$\begin{array}{l}\text { Projected } 252 \\
\text { Days from Eq }\end{array}$} & \multirow[b]{3}{*}{$\begin{array}{l}\text { Projected } 365 \\
\text { Days from Eq }\end{array}$} \\
\hline & & & & & & & & \\
\hline & & $\begin{array}{l}\text { After 7 Days } \\
\text { fro Eq }\end{array}$ & $\begin{array}{l}\text { Projected } 10 \\
\text { Days from Eq }\end{array}$ & $\begin{array}{l}\text { Projected } 30 \\
\text { Days from Eq }\end{array}$ & & & & \\
\hline$y=0.0031^{*}(x)+639.23$ & Handle Count & 639.2517 & 639.261 & 639.323 & 639.509 & 639.788 & 640.0112 & 640.3615 \\
\hline$y=3131.9^{*}(x)+2 E+09$ & Private Bytes & 2000021923 & 2000031319 & 2000093957 & 2000281871 & 2000563742 & 2000789239 & 2001143144 \\
\hline$y=0.0009 *(x)+46.67$ & Thread Count & 46.6763 & 46.679 & 46.697 & 46.751 & 46.832 & 46.8968 & 46.9985 \\
\hline$y=6534.9^{*}(x)+2 E+09$ & Working Set & 2000045744 & 2000065349 & 2000196047 & 2000588141 & 2001176282 & 2001646795 & 2002385239 \\
\hline
\end{tabular}

Results of Reliability Test: The systems on this graph were analyzed in a reliability test. Analyzed the graphs in this research and take the necessary action on it. This action depends on the following conditions.

1) When Graph is flight mode, then assume system is working fine.
2) When Graph is continuously increasing mode, then assume system is lagging or some problem occurred.

3) When Graph is continuously decreasing mode, then assume system is lagging or some problem occurred.

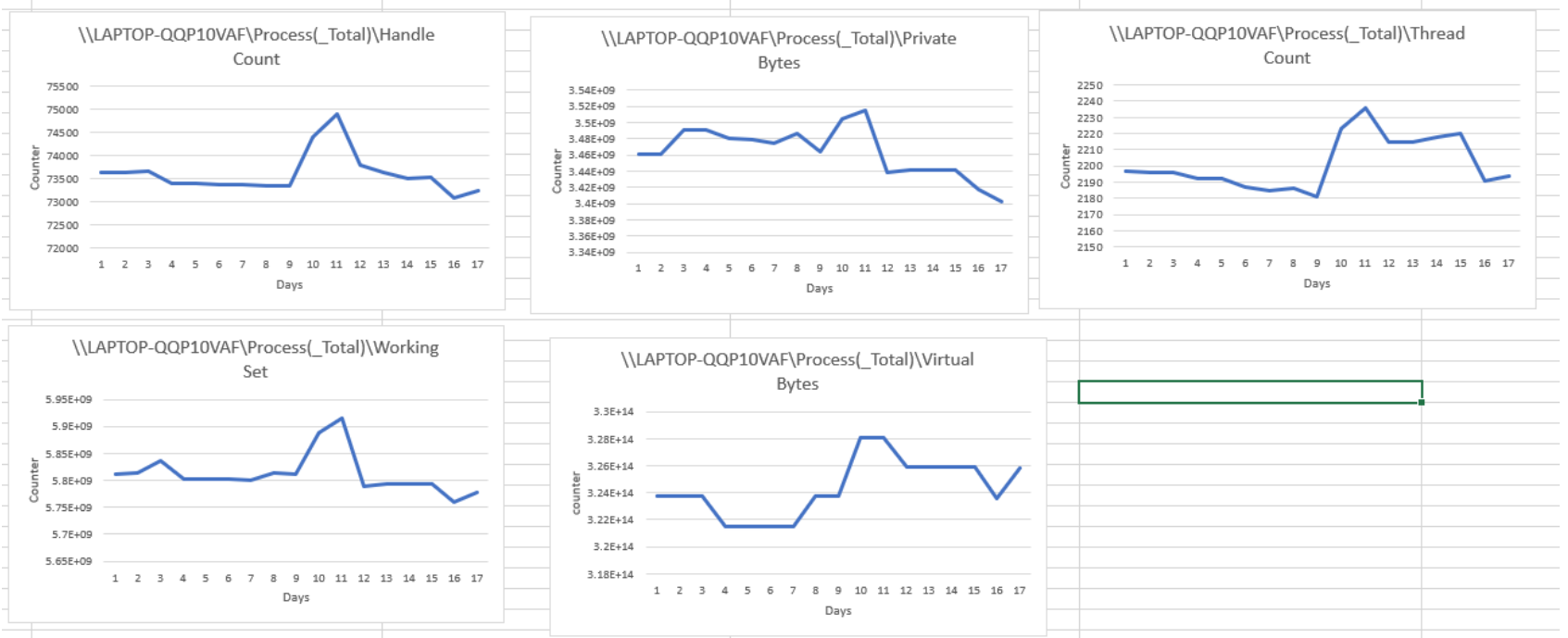

Figure 8: Reliability Test Graph

\section{Conclusion}

Performance and Reliability analysis has always been a great challenge for developers and QA engineers. In this study proposed and demonstrated real-time instruction tracing capabilities of using JMeter Tools and Performance Monitor Tools. Calibration Web Application need to be critically evaluated for their performance in real time scenarios. A logical investigation of devices as far as their task's dependent on a few boundaries like response time is portrays in the current exploration paper. The performance of both the tools has done on sample web services and web pages using different number of users and their results are tabulated on the basis of the parameters.

\section{Acknowledgments}

We express our sincere gratitude to all the authors for inspiring research attempts by virtue of their publications. We are thankful to all the authors whose papers are referred for preparing this article.

\section{References}

[1] https://www.Calibration.com/measurementsensing/ pressure measurement/test-and-calibration, calibrationasset management software

[2] https://classictechnology.ie/wpcontent/uploads/2018/10 /4sight2- datasheet.pdf

[3] https://www.capterra.com/p/172598/4Sight2 Calibration Asset Management.

[4] https://en.wikipedia.org/wiki/Calibration.

[5] https://www.edureka.co/blog/jmetertutorial/text=ApacheJmetertools.

[6] https://ieeexplore.ieee.org/document/8806309/Researc $\mathrm{h}$ on Performance Automation Testing Technology Based on JMeter.

[7] https://ieeexplore.ieee.org/document/8748521/Perform ance Analysis of Automated Testing Tools: JMeter and Test Complete.

[8] https://ieeexplore.ieee.org/document/5609969/JMeterbased aging simulation of computing system.

[9] https://ieeexplore.ieee.org/document/7780234?section= abstract/Study of Performance Testing of Information System Based on Domestic CPU and OS.

[10] https://www.site24x7.com/application-performancemonitoring. 
[11] https://techcommunity.microsoft.com/t5/ask-theperformanceteam/ windows-performance-monitoroverview/ba-p/375481

[12] https://ieeexplore.ieee.org/document/Experiences in performance testing of web applications with Unified Authentication platform using Jmeter

[13] https://ieeexplore.ieee.org/document/Performance inquisition of web services using soap UI and JMeter
[14] https://www.sciencedirect.com/topics/computerscience/performancemonitor.

[15] https://www.researchgate.net/publication/Hardware Performance Monitoring for the Rest of Us A Position and Survey.

[16] https://www.sciencedirect.com/science/article/pii/Perfo rmance monitor 